\title{
...votre pensée, s'enlaçant à la fiction. Ry/Bovary, une médiation littéraire grand public
}

...Your Thoughts, Intertwined With the Fiction. Ry/Bovary, a Literary Mediation for the General Public

Jean-Marie Privat

\section{OpenEdition}

\section{Journals}

Édition électronique

URL : https://journals.openedition.org/recherchestravaux/2157

DOI : 10.4000/recherchestravaux.2157

ISSN : 1969-6434

Éditeur

UGA Éditions/Université Grenoble Alpes

Édition imprimée

ISBN : 978-2-37747-197-3

ISSN : 0151-1874

\section{Référence électronique}

Jean-Marie Privat, « ...votre pensée, s'enlaçant à la fiction. Ry/Bovary, une médiation littéraire grand public », Recherches \& Travaux [En ligne], 96 | 2020, mis en ligne le 23 juin 2020, consulté le 30 juin 2021. URL : http://journals.openedition.org/recherchestravaux/2157 ; DOI : https://doi.org/10.4000/ recherchestravaux.2157

Ce document a été généré automatiquement le 30 juin 2021.

(C) Recherches \& Travaux 
...votre pensée, s'enlaçant à la fiction. Ry/Bovary, une médiation littéraire grand public

... Your Thoughts, Intertwined With the Fiction. Ry/Bovary, a Literary Mediation for the General Public

Jean-Marie Privat

1 Un lieu, un personnage, un dispositif. Le lieu c'est Ry, petit village à quelques lieues de Rouen. Le personnage c'est Emma, son encrage/ancrage dans le paysage normand. Le dispositif c'est un dispositif local de célébration culturalo-touristique d'une héroïne de roman. Aussi est-ce une ethnographie des pratiques littéraires que nous nous proposons de documenter ici pour illustrer en quelque façon une anthropologie de l'imaginaire contemporain, in situ.

\section{... au pays qui nous ressemble/rassemble}

2 Madame Bovary, mœurs de province (1857) se prête aisément à une lecture disons «réaliste». Dès sa publication, Flaubert dut protester contre les rapprochements biographiques qu'une partie de son lectorat - bienveillant - s'ingéniait à établir entre tel pharmacien et M. Homais, telle petite femme de docteur de campagne et son héroïne de papier :

Non, Monsieur, aucun modèle n'a posé devant moi. Madame Bovary est une pure invention. Tous les personnages de ce livre sont complètement imaginés, et Yonville-l'Abbaye lui-même est un pays qui n'existe pas, ainsi que la Rieulle, etc. Ce qui n'empêche pas qu'ici, en Normandie, on n'ait voulu découvrir dans mon roman une foule d'allusions ${ }^{1}$.

Et ce mode de lecture à l'identification référentielle (à l'identifiction si l'on me passe le mot) fut l'objet même d'une certaine presse régionale distinguée qui dès la mort de l'auteur partit en quête des "témoins" locaux qui acceptaient sans l'ombre d'une difficulté d'entrer dans cette logique du modèle réaliste de la fiction et de ses sources². 
Il s'engagea même au début du siècle dernier une querelle assez vive - sur fond de développement d'une première forme de tourisme littéraire - sur le fait de savoir quels étaient les "lieux » véritables d'un récit si manifestement normand'. C'est le petit village de Ry - sa longue rue, sa rivière paresseuse, son auberge, ses halles, sa mairie, etc. - qui s'imposa comme le modèle de référence de Yonville ${ }^{4}$. L'argument décisif reposait sur un sophisme ou un paralogisme impeccable redoublé d'un jeu d'analogie verbale. En effet la preuve formelle que Ry était Yonville et que Yonville était bel et bien le village des époux Bovary c'est que la correspondance du toponyme et de l'anthroponyme est codée dans les mots eux-mêmes: Ry est nécessairement à BovaRy ce que BovaRy est à Ry. Bref, Bovary c'est Ry. Tout est dit et écrit ${ }^{5}$. Ce jeu avec l'homonymie partielle - proche de la devinette - est certes le signe d'un abandon facile au complaisant mirage du langage, un peu comme dans le roman le jeu de mots populaire et hospitalier sur Au Lion d'or / Au lit on dort. Mais en fait cette logique approximative du signifiant est plus intéressante qu'un banal effet de réel. Le piège de l'illusion référentielle ne se referme pas en effet sur n'importe quelle association de mots. Ainsi pour Tostes, le nom du premier village dans lequel vivent les époux Bovary. Il est facile de vérifier sur une carte que le Tostes du roman correspond sans difficulté au Tôtes de la réalité. Mais précisément c'est parce que cette adéquation entre le nom d'un lieu romanesque et le nom d'un lieu réel ne fait ni problème ni débat que peut s'entrouvrir l'espace interstitiel d'un imaginaire. En effet, l'horizon d'attente du lecteur ordinaire n'est pas d'ordre strictement géographique. C'est le roman de la réalité que ce lecteur veut entendre, la réalité comme romance tragique même et sûrement pas la réalité du roman comme empire des signes et comme style. Ainsi s'actualise un imaginaire topique, imaginaire à la fois toponymique et anthroponymique. Ainsi se crée un dispositif verbal et mental en anamorphose où le pays (normand) se mue en paysage (littéraire) et le paysage en page d'écriture ; et dans le même temps se déploie sur la page un paysage d'un petit pays. Madame Bovary, mours de province, au pays qui nous ressemble \& rassemble ${ }^{6}$.

4 Il faut dire que le texte du roman se prêtait lui-même assez volontiers à cette implication du lecteur dans la fiction. Ici, il sera question de « l'amitié douce de quelque bon petit frère, qui va chercher pour vous des fruits rouges dans des grands arbres plus hauts que des clochers ${ }^{7} »$. Ailleurs, la nourrice est « si triste, si triste, qu'à la voir debout sur le seuil de sa maison, elle vous faisait l'effet d'un drap d'enterrement tendu devant la porte ». Une autre fois, le pré des Comices commence à se remplir « et les ménagères vous heurtaient avec leurs grands parapluies, leurs paniers et leurs bambins ». Emma elle-même " a des yeux qui vous entrent au cœur comme des vrilles ", etc. Cette Emma qui se prend volontiers au jeu/piège du continuum entre biographème personnel et lecture intime :

Le souvenir du vicomte revenait toujours dans ses lectures. Entre lui et les personnages inventés, elle établissait des rapprochements 8 .

Ce régime réaliste de la fiction (un effet imaginaire de réel si l'on veut) et de son appropriation subjective signifie combien la littérature peut être à l'œuvre dans l'espace public ${ }^{9}$ et combien l'expérience référentielle peut donner vie à la littérature. Aujourd'hui à Ry comme sur les remparts de Francfort, jadis :

Il y avait là une niche, une fontaine, une inscription enchâssée dans le mur, et derrière le mur s'élevaient de vieux noyers. Le conte ajoutait une porte mystérieuse [...]. Le sujet fabuleux s'entrelaçait à la réalité visible et semblait jaillir de ce vieux « Mauvais Mur» entouré de légendes avec sa fontaine [...]. Les jeunes 
auditeurs de Goethe s'y rendaient en pèlerinage. Le conte avait ainsi initié une sorte

de « légende locale » et il s'était formé une sorte de « culte local $»^{10}$.

6 Si Ry rime avec Bovary, Bovary c'est bien sûr en tout premier lieu Emma Bovary. Or, le parcours fictionnel du personnage principal conjugue l'insertion dans une réalité quotidienne assez communément partagée (les mœurs de province, le fait divers villageois, les travaux, les jours et les nuits, les relations entre hommes et femmes, l'argent et le désir, les paysans et les bourgeois, la coutume et le Code, les usages sociaux et leurs transgressions, etc.) et la fin tragique d'un destin de femme.

7 Les instances locales jouent aujourd'hui de cette double donne en focalisant sur la vie et la mort de l'héroïne locale alors que les instances culturelles plus classiques et plus lointaines s'intéressent à l'écrivain et à son travail, sa langue, son style, son écriture, son ironie. À Ry, le visiteur est invité à une sorte d'immersion fictionnelle dans le monde d'Emma (la maison du pharmacien, la mairie, l'église, le chemin vers le château de son amant, la tombe de Delphine Couturier alias Emma B., etc.), une authentique feintise ludique où les lieux et le récit se font écho et se garantissent l'un l'autre. La plus-value symbolique tient alors au fait que le roman de Flaubert est certes un bien culturel légitime mais que dans la logique de la culture moyenne l'accessibilité à l'œuvre est comme facilitée par une série de médiations concrètes. Le lieu est le lien.

8 On est ainsi au plus loin de la lecture silencieuse, solitaire et méditative sur un mode académique et distancié ; et sur fond d'une certaine bonne volonté culturelle, au plus près d'une forme d'acculturation soft, passagère et paysagère, à la grande littérature.

\section{Appropriation scolaire et appropriation patrimoniale}

9 Les deux dispositifs principaux d'animation culturelle pour un public élargi sont La Galerie Bovary et la Promenade au pays d'Emma Bovary.

* La Galerie est située à l'entrée du village dans un vieux pressoir du XVIII ${ }^{\mathrm{e}}$ siècle qu'un horloger local a naguère restauré et réaménagé pour présenter sous forme de saynètes quelques-unes des scènes principales du roman ${ }^{11}$. Chacune de ces citations [comme des extraits de lecture dans les manuels] est en quelque façon une animation littéraire où des personnages miniatures et automates donnent vie à l'entrée de Charles au collège, au repas de noce à la ferme des Bertaux, aux Comices agricoles, aux escapades d'Emma à Rouen ou encore à sa mort. 
Fig. 1

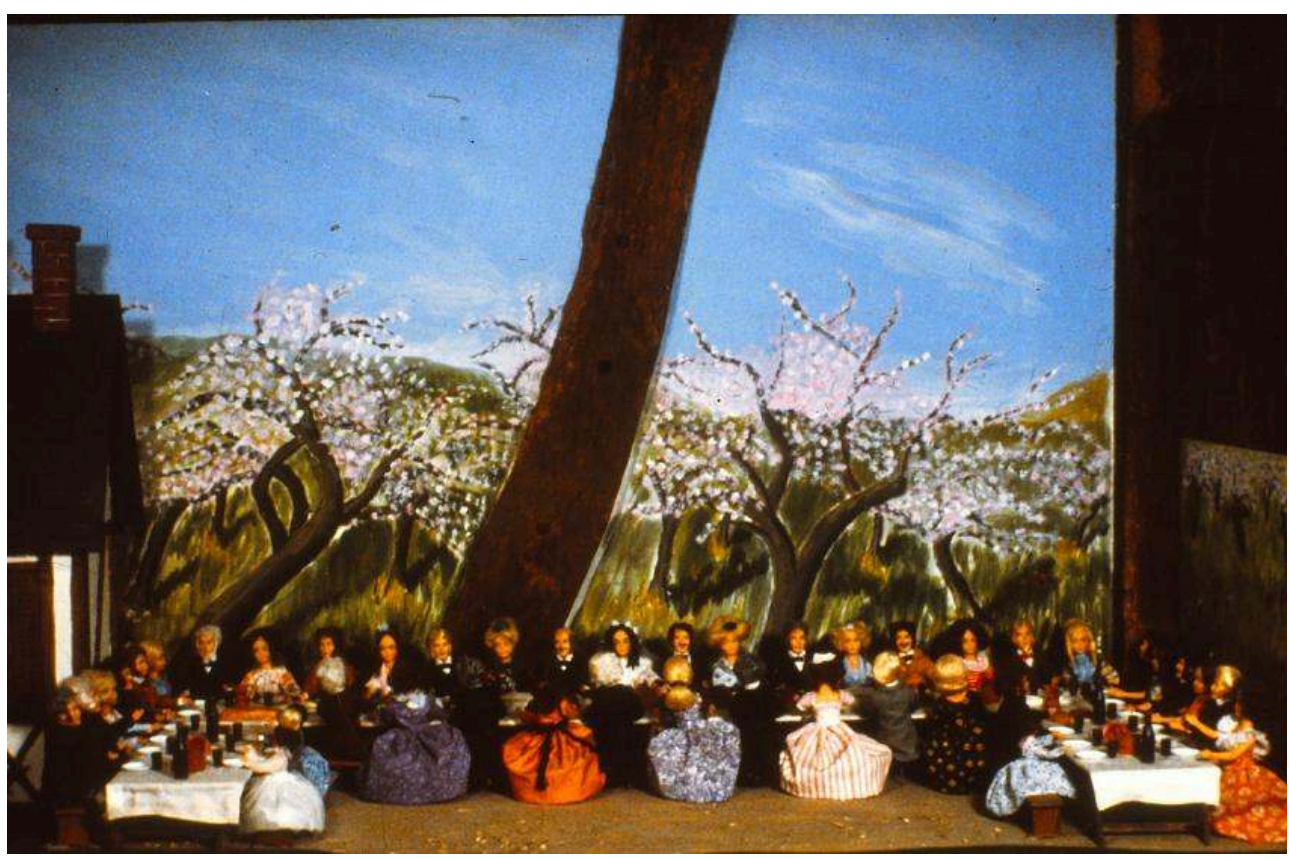

La noce aux Bertaux, Galerie Bovary. Musée d'automates. En ligne <http://musee-bovary.net/ scenesanimeesduroman.html> (consulté le 5 janvier 2020).

(c) Michel Burgaud.

Fig. 2

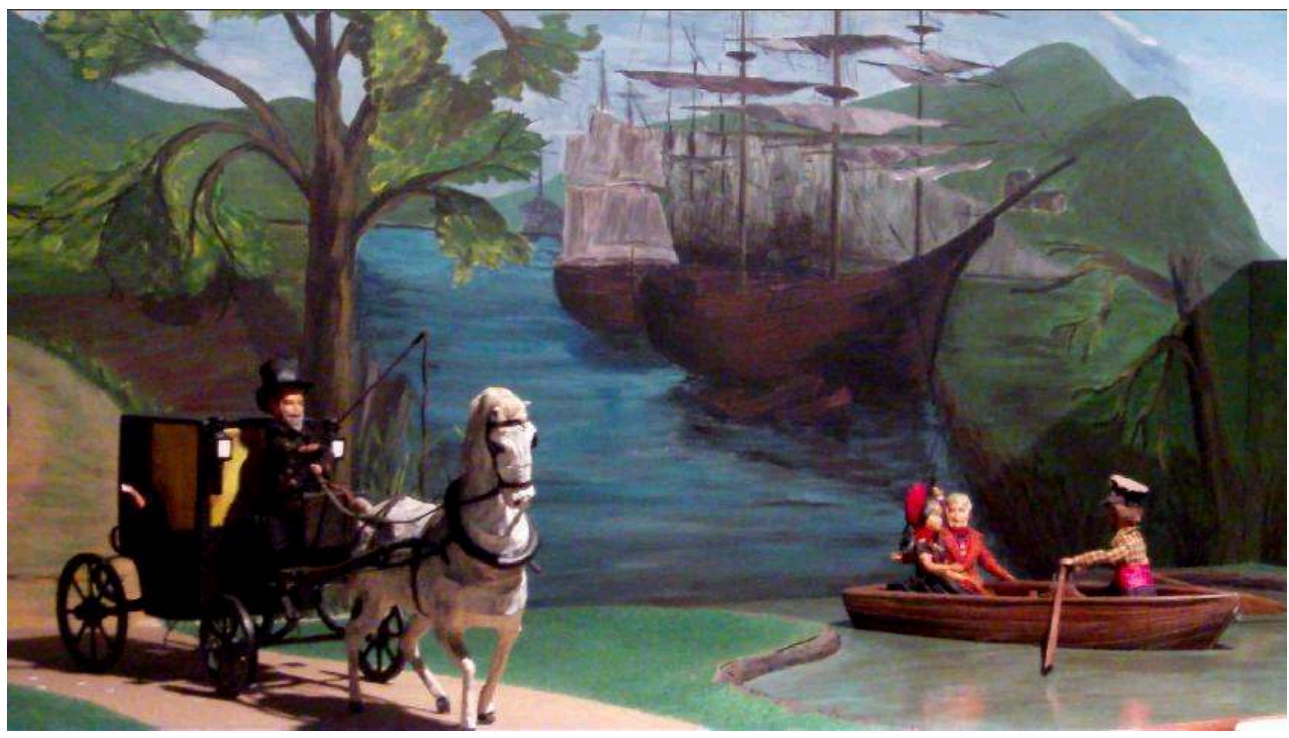

Les amants à Roue, Galerie Bovary. Musée d'automates. En ligne <http://musee-bovary.net/ scenesanimeesduroman.html> (consulté le 5 janvier 2020).

(c) Michel Burgaud.

11 Comme l'indique le prospectus de présentation, ces maquettes animées où tout est artisanal - particulièrement les 300 automates - sont conçues pour la détente de tous « littéraires, étudiants, vacanciers et familles ». Ce « clin d'œil sur les grandes scènes du roman ${ }^{12}$ » présuppose un usage socio-scolaire de la fiction, entre reconnaissance 
consensuelle du chef-d'œuvre littéraire et mode de fréquentation collective et ludique d'un petit monde encore proche, où - comme on dit - on peut s'y reconnaître ${ }^{13}$ :

Je pousserai tout grand l'auvent de la fenêtre contre le mur : vous pourrez le voir par derrière, en vous penchant sur la haie ${ }^{14}$.

On pourrait même s'y voir :

Vous êtes dans un jardin public, je suppose; un quidam se présente, bien mis, décoré même, et qu'on prendrait pour un diplomate ; il vous aborde ; vous causez; il s'insinue, vous offre une prise ou vous ramasse votre chapeau. Puis on se lie davantage; il vous mène au café, vous invite à venir dans sa maison de campagne $[. . .]^{15}$.

12 Le guide de son côté joue volontiers de cette illusion de proximité référentielle et des émotions partagées pour les heurs et malheurs des personnages principaux. Et Emma elle-même n'était pas étrangère à cet effet de microcosme et à cette scénographie mise en abyme :

Dans l'après-midi, quelquefois, une tête d'homme apparaissait derrière les vitres de la salle [...]. Une valse aussitôt commençait, et, sur l'orgue, dans un petit salon, des danseurs hauts comme le doigt, femmes en turban rose [...], singes en habit noir, messieurs en culotte courte, tournaient, tournaient entre les fauteuils [...], se répétant dans les morceaux de miroir que raccordait à leurs angles un filet de papier doré. L'homme faisait aller sa manivelle, regardant à droite, à gauche et vers les fenêtres [...]. Des sarabandes à n'en plus finir se déroulaient dans sa tête [...] ${ }^{16}$.

On songe à ce qu'esquissait Roland Barthes du «dispositif topologique » qui donne à lecture « son tracé et sa liberté »:

J'imagine assez bien le récit lisible [...] sous les traits de l'une de ces figurines subtilement et élégamment articulées dont les peintres se servent (ou se servaient) pour apprendre à croquer les différentes postures du corps humain [...]. En lisant nous imprimons une certaine posture au texte, et c'est pour cela qu'il est vivant ${ }^{17}$.

* La Promenade au pays d'Emma Bovary est un second dispositif, plus récent, qui s'inscrit très explicitement dans l'économie du développement patrimonial local. 
Fig. 3.1

Étapes 1 et 2-RY (panneaux place Flaubert et à l'église)

Avec sa rue principale s longue d'une portée de fusil et bordée de quelques boutiques $\%$, Ry

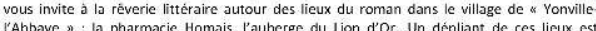
disponible à Poffice de tourisme.
La Galerie Bovary - Musée d'utomates est principalement consacrée au roman (ouvert uniquement pour les groupes sur réservation au 0235236144 )
Ne quittez pas RY sans admirer le magnifique porche Renaissance de Péglise (XVIe s.).

Étapes 3 et 4 - BLAINVILLE-CREVON (panneau au château route de Buchy)

Une route pittoresque longe le cours sinueux du Crevon, « la Rieule » dans le roman. Garezdu château médiéval qui accueille chaque année le festival \& Archéo-Jazz \%. A côté, un wétroit sentier serpentant entre les blés vertsw conduisait à la ferme Couturier. C'est dans la collégiale que se marierent Eugene Delamare et Delphine Couturier. A côté se trouve to

Étape 5 - SAINT-GERMAIN-DES-ESSOURTS (panneau près des cressonnières)

Ce village tire son nom des nombreuses sources dont les eaux vives alimentent une cressonnière. C
dans le roman.

Étape 6 - LES AUTHIEUX, SAINTE-CROIX-SUR-BUCHY (près de la chapelle)

Au hameau des Authieux, la chapelle qui associe harmonieusement grès, brique, silex et

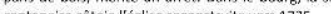

Étape 7 - BUCHY (panneau à côté de l'église)

Le bourg de Buchy, cité dans "Madame Bovary " offre la possibilité de ressentir ratmosphere des comices agricoles. En effet, chaque lundi matin se tient sous les halles en

Étape 8- BOIS-HÉROULT (panneasy en face de l'́glise)

Au milieu d'un parc qui ouvre ses portes au public, le château du début du Xville siècle et ses

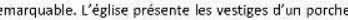
frmé en chapelle baptismale.

tape 9 - BOIS-GUILBERT (panneau en face du château)

Rendez-vous devant le chateau ou les amoureux de la nature et de la sculpture pourront France et ses quelques 200 poneys accueilent les amateurs de randonnee equestr.

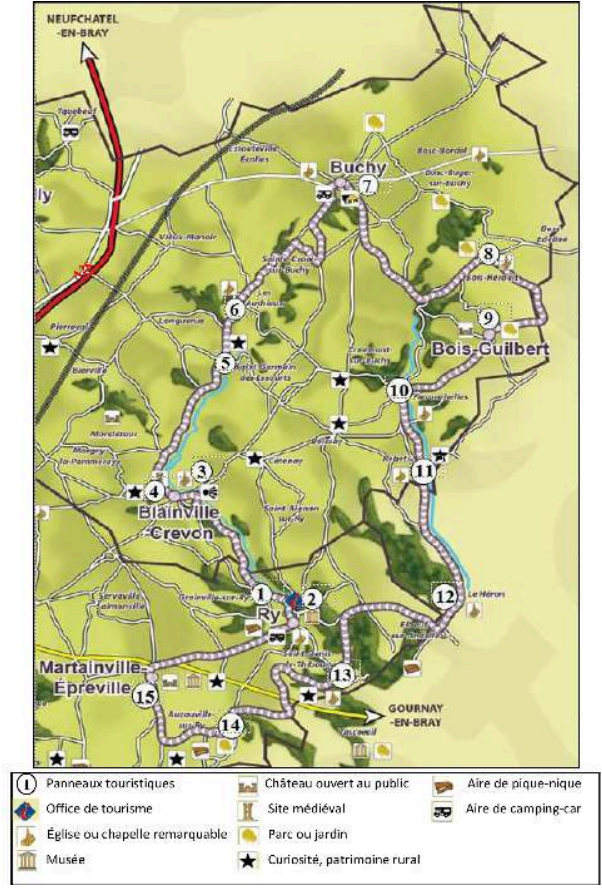

Dépliant 1 (recto) «Promenade au pays d'Emma Bovary », office de tourisme des Trois Vallées à Ry. En ligne : <https://cdt76.media.tourinsoft.eu/upload/Depliant-Promenades-au-pays-d-EmmaBovary.pdf> (consulté le 5 janvier 2020).

Fig. 3.2

Etape 10 - HÉRONCHELLES (panneau en face de l'église)

Ici naquit François Thérain, aubergiste et conducteur de la diligence à Ry, qui évoque le « Père Hivert " et son \&

Étape 11 - REBETS (panneau en face de 'église)

Le détour par ce villağe qui domine la vallée permet de découvrir, en montant sur les hauteurs, le magnifique parche en pierre (1547) de l'église.

Étape 12 - LE HÉRON (panneau en face des ruines de l'église)

C'est au chăteau du marquis de Pomereu que le jeune Gustave Flaubert prit conscience, comme Emma, des fastes de la vie mondaine, qui lui inspira l'épisode du bal de « La Vaubyes. sard \%. De lancien domaine subsistent le parc dessiné par Le Nơtre, le colombier et les

Étape 13 - SAINT-DENIS-LE-THIBOULT (pannezu à côté de l'élise)

La route qui vous mene au village offre un superbe point de vue sur la vallée du Crevon. Vous pourrez voir de belles constructions en brique : un château, un moulin et la chapelle funéra
Saint-Laurian. Au Hameau de Villers, la propriété de la Huchette aurait accueilili les rend vous galants d'Emma Bovary.

Étape 14 - AUZOUVILLE-SUR-RY [panneau à cỏté du four à pain'

Ce village fut réputé, au XIXe siècle pour son industrie florissante de robinets de tonneaux en (avvage près de la marc.

Etape 15 - MARTAINVILLE-EPREVILLE (panneau parking du chàteau)

Le cháltau construit en 1485 abrite le musée des Traditions et Arts normands : armoires belle évocation de la vie quotidienne normande au siècle de Gustave Flaubert.
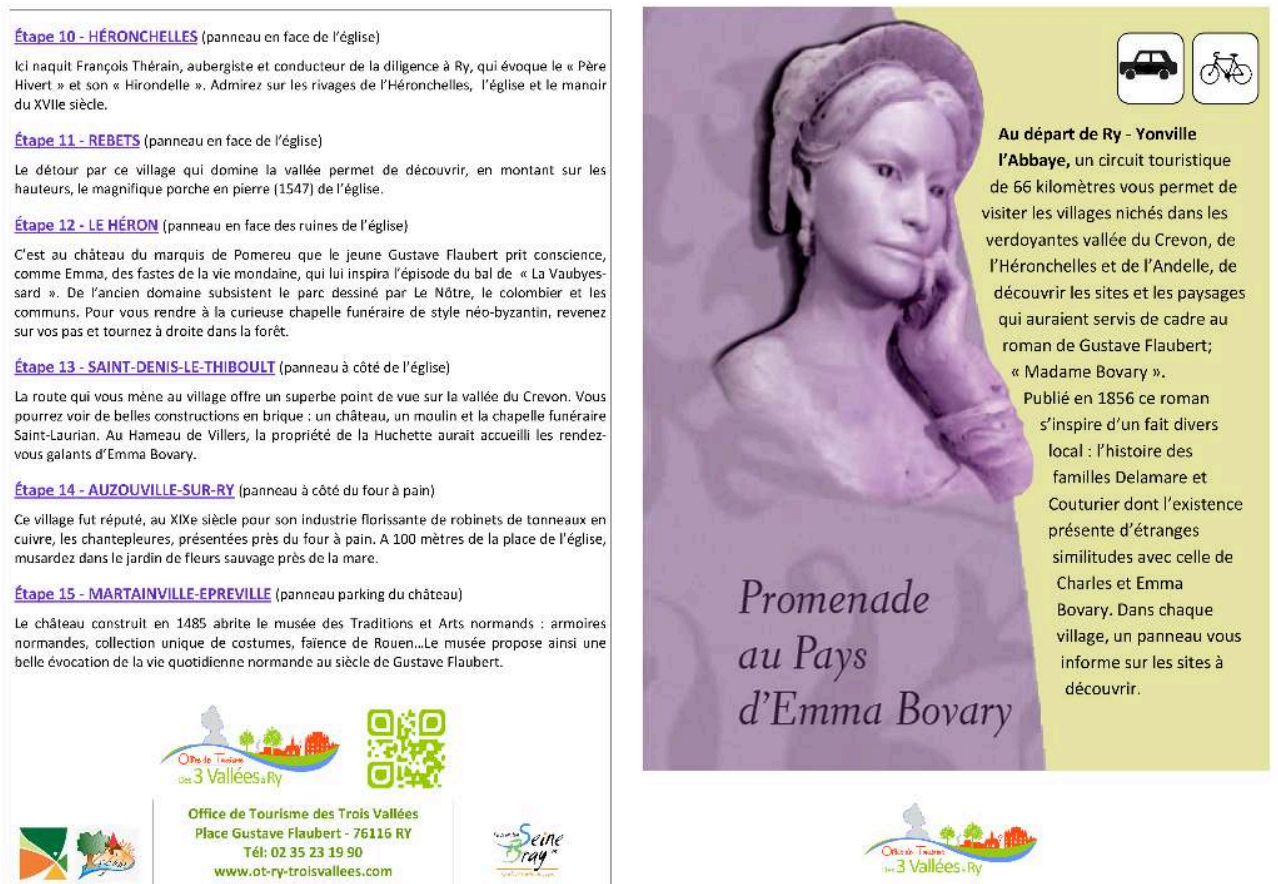

Dépliant 1 (verso) " Promenade au pays d'Emma Bovary », office de tourisme des Trois Vallées à Ry En ligne : <https://cdt76.media.tourinsoft.eu/upload/Depliant-Promenades-au-pays-d-EmmaBovary.pdf> (consulté le 5 janvier 2020). 
Le capital symbolique que représente la célèbre héroïne de Flaubert et le redéploiement in situ de son histoire offrent en effet aux visiteurs la possibilité de s'approprier un patrimoine lettré où l'imaginaire personnel peut faire son école buissonnière.

Déplions le prospectus diffusé par l'office de tourisme des Trois Vallées à Ry. Il propose en ces termes une randonnée sur les pas de Madame Bovary:

$\mathrm{Au}$ départ de Ry-Yonville, un circuit touristique de 66 kilomètres vous permet de visiter les villages nichés dans les verdoyantes vallées de Crevon, de l'Héronchelles et de l'Andelle, de découvrir les sites et les paysages qui auraient servi de cadre au roman de Gustave Flaubert. Publié en 1856 ce roman s'inspire d'un fait divers local : l'histoire des familles Delamare et Couturier dont l'existence présente d'étranges similitudes avec celle de Charles et Emma Bovary. Dans chaque village, un panneau vous informe sur les sites à découvrir ${ }^{18}$.

Fig. 4

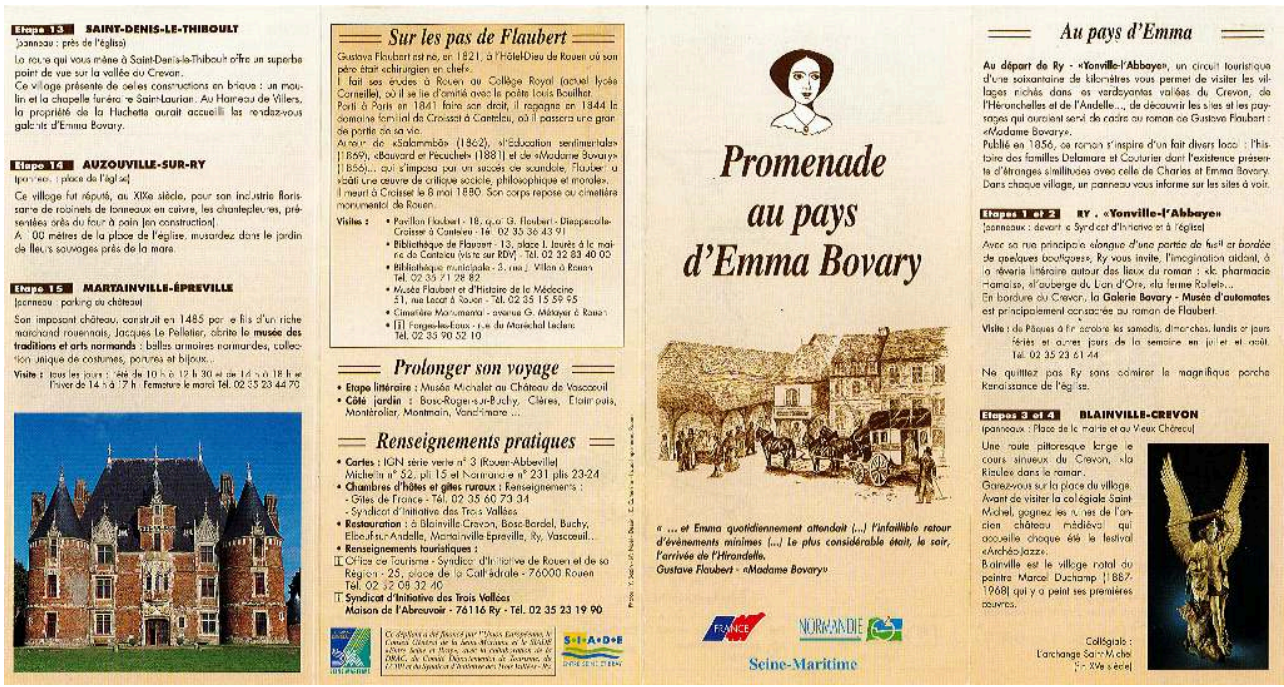

Dépliant 2 «Promenade au pays d’Emma », office de tourisme des Trois Vallées à Ry.

16 On le voit, le visiteur est invité à mettre ses pas dans un univers où il est difficile de démêler le fait divers de l'univers littéraire, la légende topique de la romance de papier. Cet enchevêtrement des régimes de réalité participe sans doute d'un plaisir culturel qui tient à distance les catégories discursives savantes et laisse libre cours à une quête de mimésis. On peut lire par exemple sur les premiers panneaux que Ry avec «sa rue principale "longue d'une portée de fusil et bordée de quelques boutiques" invite à la rêverie littéraire autour des lieux du roman (la pharmacie Homais, l'auberge du Lion d'Or) ». À vrai dire, rêverie (ou ironie) mise à part, Flaubert suggérait une découverte « touristique » du village sur un mode énonciatif fort proche :

$\mathrm{Au}$ bas de la côte, après le pont, commence une chaussée plantée de jeunes trembles, qui vous mène en droite ligne jusqu'aux premières maisons du pays. Elles sont encloses de haies, au milieu de cours pleines de bâtiments épars [...]. Il n'y a plus ensuite rien à voir dans Yonville. La rue (la seule), longue d'une portée de fusil et bordée de quelques boutiques, s'arrête court au tournant de la route. Si on la laisse sur la droite et que l'on suive le bas de la côte Saint-Jean, bientôt on arrive au cimetière ${ }^{19}$.

17 Au gré de son circuit, le promeneur découvrira le petit village de Saint-Germain-desEssarts qui « tire son nom des nombreuses sources dont les eaux vives alimentent une cressonnière ». Et il apprendra que «c'est ici que naquit Augustine Ménage, la servante 
des Delamare, "Félicité" dans le roman ». Un autre panneau signalera plus loin que «la route qui mène au village de Saint-Denis-le-Thiboult offre un superbe point de vue sur la vallée du Crevon [...], la Rieule du roman ». Le même panonceau précise qu'au Hameau de Villers, «la propriété de la Huchette aurait accueilli les rendez-vous galants d'Emma Bovary ». C'est toujours ce jeu cognitif du possible et du plausible, du vraisemblable et de l'imaginable, qui est suscité, suggéré. On sera invité plus loin à faire étape à Héronchelles "où naquit François Thérain, aubergiste et conducteur de diligence qui évoque le père Hivert du roman et son Hirondelle ». Et revoilà Emma :

Elle aperçut au loin, tout au fond de l'horizon, la vieille diligence l'Hirondelle, qui descendait lentement la côte des Leux [Loups], en traînant après soi un long panache de poussière. C'était dans cette voiture jaune que Léon, si souvent, était revenu vers elle [...]. Elle crut le voir en face, à sa fenêtre, puis tout se confondit ${ }^{20}$.

18 Au pays d'Emma la fiction verbale se fond tout au long du parcours dans le pittoresque d'un paysage rustique et le petit pays de carte postale devient le décor réel de l'intrigue romanesque. Il serait (presque) impossible de s'y méprendre ${ }^{21}$. C'est ainsi qu'une proposition de rêverie littéraire peut être l'horizon d'une promenade romantique où un petit pays est investi par un monde imaginaire et où un modeste patrimoine rural se transmue ici et là en l'espace de déploiement d'une fiction lettrée. Une forme de familiarité tranquille sinon de connivence avec La Littérature. Et avec les rêveries d'Emma, entre poésie et nostalgie :

Elle allait jusqu'à la hêtraie de Banneville, près du pavillon abandonné qui fait l'angle du mur, du côté des champs [...]. Il arrivait parfois des rafales de vent, brises de la mer qui, roulant d'un bond sur tout le plateau du pays de Caux, apportaient, jusqu'au loin dans les champs, une fraîcheur salée [...]. Emma serrait son châle contre ses épaules et se levait $[. .].]^{22}$.

À mesure qu'elle avançait, elle reconnaissait les buissons, les arbres, les joncs marins sur la colline, le château là-bas [...]. Un vent tiède lui soufflait au visage [...]. Elle entra, comme autrefois, par la petite porte du parc, puis arriva à la cour d'honneur, que bordait un double rang de tilleuls touffus ${ }^{23}$.

19 Mais si cette illusion intéressée - «l'essentiel n'est pas que le lit de van Gogh soit celuilà même où il est avéré qu'il dormit : tout ce qu'attend le visiteur est qu'on puisse le lui montrer ${ }^{24} »$ - est réellement investie de sentiments, de sensations et de sens ${ }^{25}$, c'est aussi par ce qu'elle repose sur des schèmes comportementaux plus anthropologiques et des modèles culturels inscrits dans la longue durée.

\section{... cette fiction vivante}

L'imagerie ou l'imagier qu'offrent au regard la Galerie Bovary et la Promenade au pays d'Emma aurait sans doute parlé à notre héroïne. Cette culture du loisir (et de complicité culturelle) valorise en effet la participation personnelle et émotionnelle aux saillances du récit. Or, c'est ce type de coopération imaginaire et de fugace gratification par corps qu'éprouve Emma quand - par exemple - un soir au théâtre de Rouen elle se mire dans le jeu/je spéculaire d'une autre :

Elle se laissait aller au bercement des mélodies, et se sentait elle même vibrer de tout son être, comme si les archets des violons se fussent promenés sur ses nerfs, qq chose de sa vie-s'agitait devant ses yeux toute cette fiction vivantes tous ces personnages inventés qui s'agitait/aient dans l'atmosphère d'un autre monde - une jeune femme de sa taille à peu près s'avança sur la scène. elle-entama sa cavatine en sol majeur. elle se plaignait d'amour. elle voulait fuit la vie. elle demandait des ailes 
pr quitter la terre et Emma sentait la même chose qu'elle -/ croyait que c'était elle qui chantait $[. . .]^{26}$.

21 La jeune Emma Rouault, au couvent à Rouen, avait d'ailleurs déjà fait l'expérience de cette rêverie artistique ou littéraire en s'attardant alors sur les gravures de quelque keepsake, le soir, au dortoir :

Maniant délicatement leurs belles reliures de satin, Emma fixait ses regards éblouis sur le nom des auteurs inconnus qui avaient signé [...] au bas de leurs pièces. Elle frémissait, en soulevant de son haleine le papier de soie des gravures, qui se levait à demi plié et retombait doucement contre la page. C'était, derrière la balustrade d'un balcon, un jeune homme en court manteau qui serrait dans ses bras une jeune fille en robe blanche, portant une aumônière à sa ceinture [...]. Les naïves, une larme sur la joue, becquetaient une tourterelle à travers les barreaux d'une cage gothique [...]. Et vous y étiez aussi, sultans à longues pipes, pâmés sous des tonnelles, aux bras des bayadères [...]. Et l'abat-jour du quinquet, accroché dans la muraille au-dessus de la tête d'Emma, éclairait tous ces tableaux du monde, qui passaient devant elle les uns après les autres, dans le silence du dortoir et au bruit lointain de quelque fiacre attardé qui roulait encore sur les boulevards ${ }^{27}$.

22 Cet effet de présence de papier de mondes imaginés - «les tableaux du monde » - se grave, ici comme là, dans un imaginaire complice. Cette dialogie active avec l'univers des mots et des images est au fond au principe même d'un rapport participatif et presque esthésique à l'œuvre et à ses personnages. Flaubert lui-même (fût-ce par ironie à l'encontre du romantisme de la pose/posture) esquisse le statut anthropologique d'une certaine attente de lecture et fait la subtile théorie de cette procuration existentielle :

Quelle meilleure chose que d'être le soir au coin du feu avec un livre, pendant que le vent bat les carreaux, que la lampe brûle ? [...] On se promène immobile dans des pays que l'on croit voir, et votre pensée, s'enlaçant à la fiction, se joue dans les détails ou poursuit le contour des aventures. Elle se mêle aux personnages; il semble que c'est vous qui palpitez sous leurs costumes. - C'est vrai! C'est vrai! disait-elle ${ }^{28}$.

23 À Ry, on se promène de facto dans un petit pays coalescent à la fiction et cet enlacement pragmatique imaginaire induit tout à la fois comme une attention flottante au punctum de certains détails et comme l'ébauche intérieure d'une possible continuation subjective des grandes lignes du récit. Il y a même chez Flaubert - en particulier dans les brouillons - une micro-phénoménologie du cheminement d'une lecture en acte, l'évocation concrète d'un glissando de la conscience le long des lignes d'écriture et d'une subjectivité qui s'imprime au fil des pages :

on ne songe à rien [...], les heures passent - de phrase en phrase, on glisse comme sur l'eau sans s'apercevoir de la route. on vagabonde on s'oublie ${ }^{29}$

lire singulière chose quelle étrange surprise que de voir ainsi dans tout un livre imprimé ce que l'on sent soi-même [...] un singulier effet de voir imprimé dans un livre quelque chose de votre cœur [...] vos sentiments les plus déliés ${ }^{30}$ quelque chose que vous ne saviez pas. on s'arrête alors, / et l'âme étonnée se considère elle-même, comme une nymphe à la chasse qui se mire dans une fontaine ${ }^{31}$

vous-est-il arrivé qqfois de frissonner de rencontrer tout à coup au bas d'une page / comme un trésor perdu dans les broussailles ${ }^{32}$

Cette empathie plus ou moins créative et buissonnante avec l'histoire racontée est rendue possible précisément par le ré-ancrage territorial de la fiction ${ }^{33}$. Mais cette réénonciation, change le statut sémiotique du récit. Les citations littérales du roman sont en effet à la fois très éparses et très courtes, minimalistes. Ce qui est illustration « hors- 
texte » dans les manuels de littérature est ici plein champ. Le texte lui est comme tenu en lisière, les catégories discursives savantes aussi. Cette distance à l'écrit et au Logos affilie ipso facto cette forme de médiation littéraire à un puissant imaginaire topique. C'est selon nous un avatar moderne d'un double héritage culturel insu mais têtu.

\section{Un légendaire topique}

La promenade littéraire en sa version artisanale et touristique inscrit les lieux légendés par l'écrit fictionnel dans un terroir qui presque par définition, comme tout écosystème

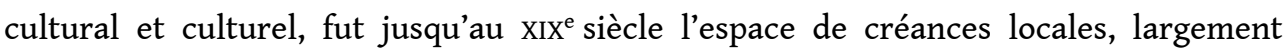
affiliée à une culture orale folklorique vivante. Le roman témoigne d'ailleurs de cette pensée sauvage $e^{34}$ qui coexiste avec d'autres cosmologies, le mode de pensée positiviste de l'apothicaire par exemple ou le romantisme rose ou funèbre d'Emma :

On [Emma et Charles] parla d'abord du malade, puis du temps qu'il faisait, des grands froids, des loups qui couraient les champs, la nuit.

$\mathrm{Ah}$ ! vous trouverez bien des préjugés à combattre, monsieur Bovary [...] car on a recours encore aux neuvaines, aux reliques, au curé, plutôt que de venir naturellement chez le médecin ou chez le pharmacien.

Ce matin même, il a fallu que j'aille [le curé du village] dans le Bas-Diauville pour une vache qui avait l'enfle, ils [les paysans] croyaient que c'était un sort. Toutes leurs vaches, je ne sais comment...

Des aboiements continus se traînaient au loin, quelque part. - Entendez-vous un chien qui hurle ? dit le pharmacien. - On prétend, qu'ils sentent les morts, répondit l'ecclésiastique. C'est comme les abeilles : elles s'envolent de la ruche au décès des personnes. Homais ne releva pas ces préjugés, car il s'était rendormi.

Le jour se leva. Il [le père Rouault, fermier] aperçut trois poules noires qui dormaient dans un arbre ; il tressaillit, épouvanté de ce présage. Alors il promit à la sainte Vierge trois chasubles pour l'église, et qu'il irait pieds nus depuis le cimetière des Bertaux jusqu'à la chapelle de Vassonville.

Elle aperçut encore une fois l'impassible château, avec le parc, les jardins, les trois cours, et toutes les fenêtres de la façade. Elle resta perdue de stupeur [...]. Le sol sous ses pieds était plus mou qu'une onde, et les sillons lui parurent d'immenses vagues brunes, qui déferlaient ${ }^{35}$.

Ce qui paraît constitutif du site littéraire est donc la réactivation symbolique de la fiction in situ, comme si l'on pouvait être présent aux territoires de la fiction et tutoyer l'histoire d'Emma, en situation, ici et maintenant. Ces soudains hic et nunc de la fiction créent un possible effet de présence [une hypotypose immersive plus ou moins assurée et assumée].

Cette re/présentation de Madame Bovary manifeste plus profondément un double transfert inscrit dans la longue durée culturelle et cultuelle de la littérature, un transfert d'oralité fabuleuse et un transfert de croyance topographique.

La culture orale ancienne est riche d'un légendaire topique où des êtres mythiques ou assimilés marquent de leurs empreintes (au propre comme au figuré) un espace familier ou proche. Les récits ou micro-récits en sont innombrables, mais du point de vue générique et anthropologique leur régime commun est d'être le fruit d'une sorte de bricolage culturel et langagier :

Entre les mythes et les contes merveilleux, se situent de micro-récits composites qui tiennent à la fois et successivement de la légende, des récits de croyance et des histoires « vraies ». Alors que les mythes ou les contes présupposent le déploiement large des structures narratives, ces micro-récits n'en présentent que des miettes 
éparses, décrivant tantôt un événement extraordinaire, tantôt un être mythique dans ses manifestations diverses ${ }^{36}$.

Nous avons vu qu'à Ry le visiteur peut aisément s'imaginer tout au long de sa flânerie littéraire mettre ses pas dans un univers mental labile où il est difficile voire sans intérêt de démêler le roman de mœurs de la romance de papier et plus encore le fait divers du mythe sorti du livre ${ }^{37}$. Un itinéraire où les moments significatifs de la vie d'Emma sont comme dispersés dans des micro-lieux, ici une rue ou un magasin de nouveautés, là le château des rendez-vous ou la pauvre maison de la nourrice. Et ces lieux, comme il est de convention sinon de règle dans le roman réaliste, sont toujours situés et nommés. Or, une des légendes topiques les plus fameuses dans l'oralité européenne est celle dite de La Vieille Morte, en ses multiples versions et motifs :

Ce récit légendaire rapporte comment une vieille femme [veuve], punie par une fée ou une sorcière pour avoir un enfant naturel, est condamnée à errer dans la montagne [...] portant une lourde pierre sur son dos. Sa longue pérégrination est marquée par des péripéties successives, qui permettent de donner une interprétation à toute une série de toponymes [...]. La Vieille passe ainsi par le Plan de l'Enfant mort, la Tombe du Chien, Écoute s'il pleut et arrive au Pont de NoieÂne [...]. Un peu plus loin, elle laisse choir sa pierre qui forme le menhir, jadis couché et aujourd'hui redressé, dit de La Pierre de la Vieille. Enfin, la Vieille exténuée meurt à la montagne de Vieille Morte ${ }^{38}$.

Fig. 5

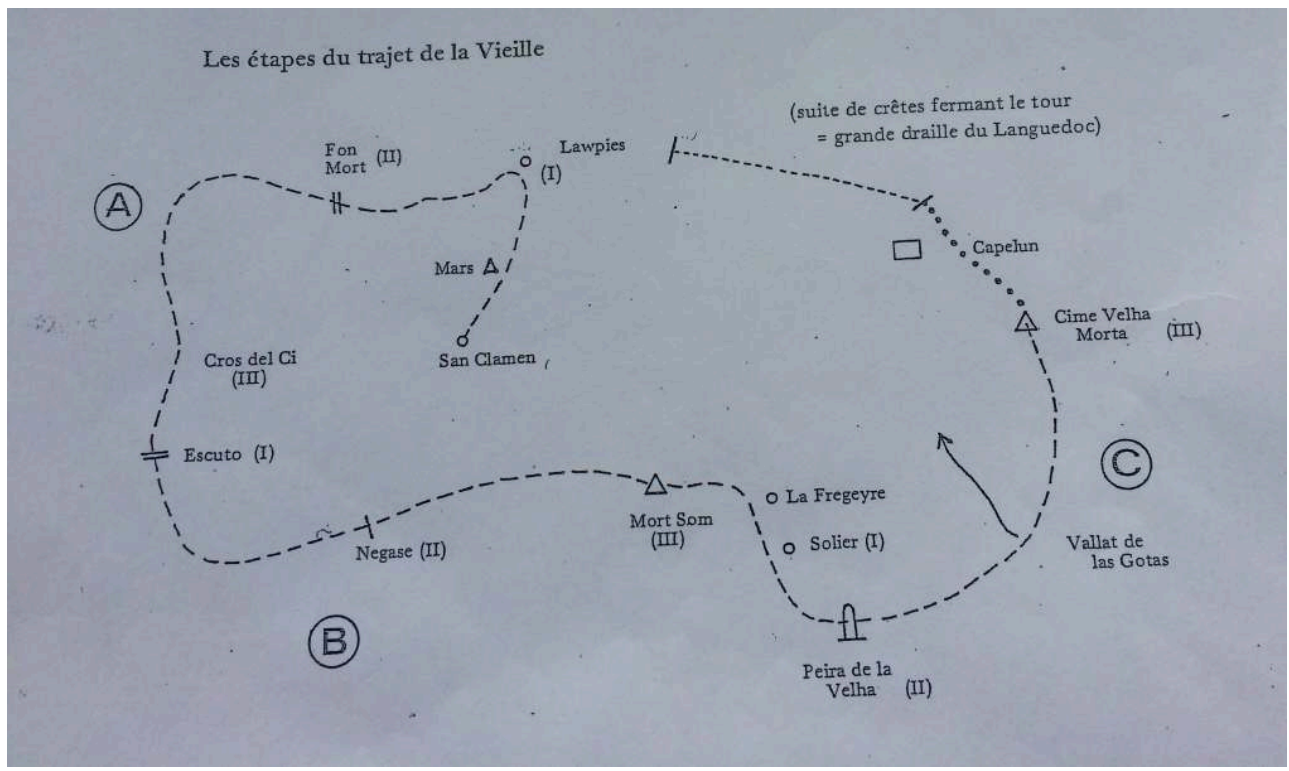

Croquis, p. 55, dans Françoise David, Michel-Marie Dufeil et Dominique Millain, « La vielha morta. Essai sur un conte cévenol », Les cahiers de Fontenay, n²3, Écrit-Oral, ENS Fontenay-aux-Roses, juin 1981, p. 43-63. Droits réservés.

30 Ces poétiques légendes étiologiques motivent les micro-toponymes qui se chargent dès lors d'une raison mythico-poétique et participent de la cosmologie locale. Les folkloristes et les anthropologues ont analysé cette dialectique générale :

La légende est un produit local [...]. Elle est vivante. Les acteurs qu'elle met en scène, chacun les connaît ou les a connus. Ce sont des gens du canton [...], c'est vous-même. Le cadre aussi est réel : vous l'avez sous les yeux. C'est le chemin creux où vous avez passé cinquante fois $[. . .]^{39}$.

Les mythes se rajeunissent dans l'histoire. Ils y puisent des éléments de réalité, qui consolident la croyance dont ils sont l'objet en tant que mythes [...]. Le 
rajeunissement des mythes n'est pas un phénomène différent du phénomène général de leur localisation dans le passé, mais une forme particulière du même phénomène $e^{40}$. Vieille Morte ait quelque rapport direct avec la cosmologie de Madame Bovary, version papier ou version locale. Il est toutefois intéressant d'observer que pour la culture orale et folklorique les toponymes sont des embrayeurs de récit et/ou sont des micro-récits en puissance. Cette cartographie fictionnelle d'un univers commun, connu et reconnu pourrait-on dire, lui donne sens. La fiction habite le terroir en donnant sens à la singularité d'un lieu et, au moins de façon latente, habite la mémoire collective. On se souvient, par exemple, qu'au Hameau de Villers, proche de Ry, «la propriété de la Huchette aurait accueilli les rendez-vous galants d'Emma Bovary». Il y a ainsi, conjointement, une situation précise de traversée du pays/paysage et une montée en mythisme et en féerie de l'élan amoureux d'Emma dans les pages du roman :

Un matin, que Charles était sorti dès avant l'aube, elle fut prise par la fantaisie de voir Rodolphe à l'instant. On pouvait arriver promptement à la Huchette [...]. Elle se trouva bientôt au milieu de la prairie, où elle marchait à pas rapides, sans regarder derrière elle.

Le jour commençait à paraître. Emma, de loin, reconnut la maison de son amant, dont les deux girouettes à queue-d'aronde se découpaient en noir sur le crépuscule pâle.

Après la cour de la ferme, il y avait un corps de logis qui devait être le château. Elle y entra, comme si les murs, à son approche, se fussent écartés d'eux-mêmes [...].

Chaque fois maintenant que Charles sortait de bonne heure, Emma s'habillait vite et descendait à pas de loup le perron qui conduisait au bord de l'eau [...]. La berge était glissante; elle s'accrochait de la main, pour ne pas tomber, aux bouquets de ravenelles flétries. Puis elle prenait à travers des champs en labour, où elle enfonçait, trébuchait et empêtrait ses bottines minces [...]. Elle arrivait essoufflée, les joues roses, et exhalant de toute sa personne un frais parfum de sève, de verdure et de grand air. Rodolphe, à cette heure-là, dormait encore. C'était comme une matinée de printemps qui entrait dans sa chambre ${ }^{41}$.

Mais, on le sait, c'est à une Emma en Jeune Morte que le roman destine son héroïne : Elle partit donc vers la Huchette [...]. Un vent tiède lui soufflait au visage [...]. Elle entra, comme autrefois, par la petite porte du parc, puis arriva à la cour d'honneur, que bordait un double rang de tilleuls touffus [...]. Les chiens au chenil aboyèrent tous, et l'éclat de leurs voix retentissait sans qu'il parût personne [...].

Elle sortit. Les murs tremblaient, le plafond l'écrasait ; et elle repassa par la longue allée, en trébuchant contre les tas de feuilles mortes que le vent dispersait. Enfin elle arriva au saut-de-loup devant la grille. [...] Elle resta perdue de stupeur, et [...] croyait entendre s'échapper comme une assourdissante musique qui emplissait la campagne. Le sol sous ses pieds était plus mou qu'une onde, et les sillons lui parurent d'immenses vagues brunes, qui déferlaient [...]. La nuit tombait, des corneilles volaient [...]. Alors sa situation, telle qu'un abîme, se représenta [...]. Elle jeta un cri aigu [...]. Elle n'existait plus ${ }^{42}$.

Comme dans la configuration légendaire toutefois, le personnage féminin du roman a transgressé au su et au vu de la communauté locale des règles coutumières liées au pouvoir patriarcal et à l'ordre sexuel conjugal ${ }^{43}$. Ce personnage principal est voué à une sorte d'itinérance dans un espace proche qui se referme progressivement sur lui/elle. Vieille et Jeune sont condamnées à une (mauvaise) mort, violente, cruelle et solitaire. On le voit, le roman opère un transfert d'imaginaire langagier selon une axiologie culturelle qui est propre à la modernité littéraire. Même si dans le récit moderne les actions et les actants sont différemment distribués ${ }^{44}$, la dissémination symbolique du 
corps d'Emma dans l'espace de la campagne normande continue de ponctuer son destin et de manifester une certaine continuité anthropologique de la mise en mot et en scène de l'insoumission des femmes et de la transgression châtiée. Comme si ces histoires s'affiliaient l'une et l'autre, et à la longue durée des genres littéraires, et à l'histoire longue des représentations du malheur comme des quêtes de bonheur(s) :

Est-ce que cette misère durerait toujours? Est-ce qu'elle n'en sortirait pas? Elle valait bien cependant toutes celles qui vivaient heureuses [...] ? Elle exécrait l'injustice de Dieu [...]. Elle s'appuyait la tête aux murs pour pleurer ${ }^{45}$.

C'est pourquoi, même si dans le discours du roman de Flaubert et sur le site littéraire de Ry il n'est pas question du destin de l'humanité mais de la destinée d'un personnage hors-norme (et la conscience qu'il a de sa différence), il y a dans la figure légendaire d'Emma - son mythe, son site - une dimension collective. La légende comme le roman fascinent sans doute car l'un comme l'autre racontent - à leur façon - ce qui se passe quand on s'écarte des conventions sociales. Ils nous parlent peu ou prou de "l'émergence d'un sujet moderne [...] qui pose les questions à ne pas poser au monde dont il se détache ${ }^{46} » \ldots$

\section{Un transfert de sacralité ?}

34 À Ry, la promenade culturelle fut très vite désignée comme un pèlerinage littéraire ${ }^{47}$. Et plus généralement les anthropologues des imaginaires littéraires in situ ont bien perçu eux aussi ce type de transfert de sacralité :

Parfois, ce qui est issu du cerveau d'un romancier [...] rencontre un tel crédit qu'on finit par oublier à peu près la nature largement sinon tout à fait fictive du thème de départ. L'endroit qui était le théâtre supposé d'une action romanesque plus ou moins imaginaire [...] se mue en lieu hautement historique, comme si cette action, devenue vraie, plongeait maintenant de solides racines dans la réalité topographique et la transfigurait ${ }^{48}$.

Le mot (plus ou moins rhétorique ?) de "pèlerinage » dit bien la croyance sinon dans l'esprit du lieu du moins le statut ontologique particulier d'un lieu habité par une fiction. Si le prestige d'une narration impulse parfois cette fiction de fiction c'est aussi parce qu'une autre infrastructure cognitive peut parfois se greffer sur la réappropriation de l'héritage topique des cultures orales ${ }^{49}$. C'est un schème d'origine religieuse qui opère cette fois dans la réappropriation symbolique et d'une fiction et d'un lieu. C'est un exemple parmi bien d'autres d'un transfert de sacralité - des saints aux héros et des héros aux héroïnes ${ }^{50}$... - qui s'est opéré progressivement du folkloricoliturgique au culturel dans le monde occidental moderne ${ }^{51}$. Comme si des fictionnèmes littéraires qui ponctuent l'imaginaire d'un espace local se substituaient maintenant à d'autres récits qui longtemps se fixèrent en des terroirs coutumiers (l'arbre aux fées, le chemin des Dames, la pierre de Saint-Pierre, le moulin du Diable, etc.), comme une exigence anthropologique partagée d'empoétisation du monde.

On a compris que le microcosme de la Galerie des automates tenait de la crèche animée, tant du point de vue de sa conception artisanale que des effets de magie ludique sur les spectateurs bons enfants. On sait assez vite quand on "enquête" à Ry que dans telle maison on conserve pieusement le "parapluie» d'Emma, sous tel hangar une «relique » de L'Hirondelle et qu'au cimetière qui jouxte l'église du village la tombe de 
Delphine Couturier/Emma Bovary est régulièrement fleurie. Et comme il se doit chaque année, pour la sainte Emma, à Ry, on peut désormais fêter dignement Emma ${ }^{52}$.

Fig. 6

\section{Fête d'Emma}

\section{Dimanche 23 avril 2006 à Ry}

A l'occasion des 150 ans de la parution du roman Madame Bovary de Flaubert

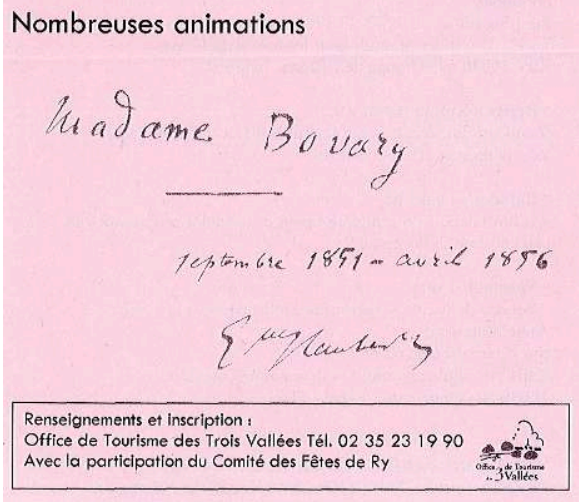

Programme de la journée

- Exposition

"Ry et Madame Bovary » par G. Besenwald à la Maison de

Entrée libre de $10 \mathrm{~h}$ à $12 \mathrm{~h} 30$ et de $14 \mathrm{~h}$ à $18 \mathrm{~h}$

$\checkmark$ Rencontres littéraires

Des auteurs régionaux présenteront et dédicaceront leurs ouvrages d

.

Entrée libre de $14 \mathrm{~h}$ à $18 \mathrm{~h}$

- Balade littéraire

Conférence suivie d'une visite à deux voix (conférencier/lecteur)

dans le bourg de Ry en compagnie de la Société des Ecrivains

normands.

normands.

Tarif: 5 adultes, $g$ ratuit pour les moins de 12 ans.

RdV 10 h30 à la Grange de l'Espace Flaubert

- Repas «Emma Bovary "

Menu spécial au restaurant La Table d' Oscar (Le Bovary) Réservation au 0235236146

- Balades en calèche

"L'hirondelle » vous attendra pour d'agréables promenades en calèche dans le bourg de Ry.

- Spectacle conté

" Secrets de femrnes, secrets de Mélusine » par la conteuse

Anne Marchand.

Sur réservation au 0235231990

Tarif : 6 adultes, $g$ ratuit pour les moins de 12 ans.

$15 \mathrm{~h} 30$ à la Grange de l'Espace Flaubert

Avec la participation du Comité des Fêtes de Ry,

du restaurant La Table d'Oscar(Le Bovary) et l'Epicuric

Office de tourisme des Trois Vallées (Ry), Fête d'Emma, programme, 23 avril 2006. on prend en compte les « témoignages » des premiers « témoins » (la bonne des Bovary, le conducteur de diligence, etc.) et des derniers visiteurs qui les uns et les autres inscrivent la geste d'Emma dans une logique topographique et légendaire en affinité anthropologique avec celle des Évangiles :

Toute mémoire [religieuse] est faite de traditions qui remontent à des évènements très éloignés souvent dans le passé, et qui se sont produits en des lieux déterminés. Il serait bien difficile d'évoquer l'événement si l'on ne songeait pas aux lieux qu'on pourrait voir [...]. On sait qu'il y a eu sur un même lieu plusieurs traditions différentes [...]. Si cependant les fidèles s'accommodent de ces variations et contradictions c'est que la mémoire [...] a besoin de se figurer les lieux pour évoquer les évènements qui s'y rattachent. Il suffit qu'ils les imaginent et qu'ils sachent qu'ils subsistent [...]. Toute l'histoire évangélique est écrite sur le sol [... ${ }^{53}$.

Si cet ancien dispositif de créance peut jouer un rôle structurant à Ry, c'est sans doute parce que l'histoire terrestre du Christ est précisément un «mélange radical de réalité quotidienne et de tragique sublime ${ }^{54}$ » et parce que plus généralement "pour se constituer dans la mémoire collective une vérité doit se présenter sous la forme concrète d'un événement, d'une figure personnelle, ou d'un lieu [... $]^{55}$ ». Ce type d'affiliation et sa reconfiguration spectaculaire et profane dans le champ littéraire local participent d'un effet de présence et d'authenticité d'autant plus puissant qu'il passe paradoxalement au filtre d'une double rêverie, « topographique et romanesque » :

Le 10 [octobre], de grand matin, je sortis de Jérusalem par la porte d'Éphraïm [...] dans le dessein d'examiner les champs de bataille immortalisés par le Tasse. Arrivé 
au nord de la ville, entre la grotte de Jérémie et les sépulcres des rois, j'ouvris $L a$ Jérusalem délivrée, et je fus sur-le-champ frappé de la vérité de l'exposition du Tasse [...]. Rien de plus net, de plus clair, de plus précis que cette description; elle eut été faite sur les lieux qu'elle ne serait pas plus exacte. La forêt placée à six milles du camp, du côté de l'Arabie, n'est point une invention du poète [...]. On est absolument sur les lieux [...]. Aladin est assis avec Herminie sur une tour bâtie entre deux portes [...]. Cette tour existe $[. . .]^{56}$.

«Enfin...", commente Halbwachs, «elle existe surtout dans l'imagination de Chateaubriand: ombre de tour et fantôme de forêt ". C'est bien un imaginaire proprement fabuleux qui commande aussi lorsque par-delà le mémorable factuel et la transaction symbolique avec les lieux du roman, le public des lecteurs et des visiteurs de Ry/Bovary n'hésitent pas à s'imaginer une fiction anthropologique qui les place en situation généalogique de parenté plus ou moins directe avec Delphine Delamare/ Emma Bovary, roman du roman :

« 26 avril 2005 - Bonjour [...]. Mon intérêt est à la fois littéraire et patronymique voire familial à un très lointain degré. Avec mes remerciements anticipés. François Delamare»

«27 octobre 2005 - Bonjour [...]. Je suis généalogiste amateur et originaire de Buchy, village proche de Ry[...]. J'ai des éléments de généalogie détaillés concernant Véronique Delphine Couturier (Mme Bovary). J'ai moi-même un cousinage avec cette Madame Bovary. Bien cordialement. Sophie D. »

« 7 juillet 2009 - Bonjour [...]. L'histoire des Delamare j'aimerais la connaître, je m'appelle moi-même Delphine Delamare 闻 57 . "

Lire/Écouter/Voir : le lecteur, l'écouteur, le promeneur sont donc des personnages de la fiction et des performeurs du récit. Nous dirions volontiers, en paraphrasant Roland Barthes, qu'ils [elle] ne décodent pas, mais qu'ils [elle] surcodent. Ils [Elle] ne déchiffrent pas. Ils [Elle] entassent des langages, ils [elle] se laissent "infiniment et inlassablement » traverser par eux : ils [elle] sont "cette traversée ${ }^{58}$ ", une traversée bio/topo/graphique :

La nuit, quand les mareyeurs, dans leurs charrettes, passaient sous ses fenêtres en chantant la Marjolaine, elle s'éveillait [...]. Et elle les suivait dans sa pensée, montant et descendant les côtes, traversant les villages, filant sur la grande route à la clarté des étoiles [...]. Elle s'acheta un plan de Paris, et, du bout de son doigt, sur la carte, elle faisait des courses dans la capitale. Elle remontait les boulevards, s'arrêtant à chaque angle, entre les lignes des rues, devant les carrés blancs qui figurent les maisons ${ }^{59}$.

\section{NOTES}

1. Lettre de Gustave Flaubert à Alfred Caillaux, Croisset, 4 juin 1857, correspondance de Flaubert, Centre Flaubert. En ligne: <http://flaubert.univ-rouen.fr/correspondance/edition> (consulté le 14 janvier 2020).

2. Se reporter entre autres à $\mathrm{R}$. Herval, Les véritables origines de Madame Bovary, Paris, Nizet, 1957 ou encore à R. Vérard, Ry, pays de Madame Bovary, Galerie Bovary Musée des automates, 1983.

3. J.-M. Privat, « Un imaginaire romanesque local », Madame Bovary, lieux réels et imaginaires, dans Bulletin des Amis de Flaubert et Maupassant, $\mathrm{n}^{\circ}$ 22, 2008, p. 57-68. Voir aussi G. Leblanc, Un pèlerinage 
au pays de Madame Bovary, E. Sansot et Cie, Paris, 1913. En ligne : <https://fr.wikisource.org/wiki/ Un_pélerinage_au_pays_de_Madame_Bovary> (consulté le 14 janvier 2020).

4. Voir par exemple, J.-M. Privat, "Emma à Ry. Notes de recherche ", ethnographiques.org, $\mathrm{n}^{\circ} 5$, avril 2004. En ligne: <https://www.ethnographiques.org/2004/Privat> (consulté le 14 janvier 2020).

5. Voir par exemple G. Venzac, Au pays de Madame Bovary. Ry ou rien, Paris, La Palatine, 1957. Comme le faisait observer jadis Paul Valéry («Au sujet d'Adonis » [1920], Études littéraires), « une consonance parfois, fait un mythe ".

6. Les petites sociétés savantes locales ont également contribué à la diffusion intéressée de cette idée des sources locales et factuelles du roman : «Par une petite route sinueuse et solitaire qui longe la vallée du Crevon, on arrive à un village dominé au Nord par un coteau au sommet duquel un rideau de hêtres cache la ferme Lepage, naguère celle du père Couturier, où grandit et fut courtisée par l'officier de santé Eugène Delamare celle qui devait être Emma Bovary - du moins, en partie [...]. À la mairie, on nous montrera le registre où, sous l'acte de mariage du 6 août 1839, s'alignent les paraphes de plusieurs originaux du roman [sic] », Gaston Bosquet, « Pèlerinage au pays de Madame Bovary ", Les Amis de Flaubert, 1960, bulletin $n^{\circ} 16$, p. 26.

7. G. Flaubert, Madame Bovary, p. 59-60. Nous citons d'après G. Flaubert, Madame Bovary. Mours de province [1857], éd. C. Gothot-Mersch, Paris, Garnier-Frères, 1971, ici p. 36.

8. Ibid.

9. Comme le fait observer D. Fabre, « la projection de la littérature dans le réel n'est pas, en soi, un fait nouveau ; on sait que quelques aristocrates du Grand Siècle se faisaient lire L'Astrée devant les paysages bucoliques du Forez ». Et dans le Berry de George Sand comme pour Proust à IlliersCombray « on va jusqu'à doubler la toponymie locale par celle que le romancier a inventée. Ces lieux littéraires et bien d'autres qui ont été "énoncés" et donc le plus souvent "refondés" par la littérature [...]", L'écrivain et ses demeures. Rapport final, ministère de la Culture et de la Communication, 2003. En ligne: <www.culture.gouv.fr/content/download/77601/589195/.../ Ethno_Fabre_2003_438.pdf>.

10. M. Bakhtine, "Le roman d'apprentissage », Esthétique de la création verbale, Paris, Gallimard, coll. «Bibliothèque des idées », 1984, p. 253.

11. Galerie Bovary. Musée d'automates. En ligne : <http://www.musee-bovary.net/index.html> (consulté le 5 janvier 2020).

12. Galerie Bovary. Musée d'automates, Ry, Michel Burgaud et fils.

13. «La confrontation de l'expérience éthico-pratique de la vie quotidienne confrontée au monde du texte est au principe de la participation, de l'identification, de l'évasion, du divertissement, qui définit la lecture littéraire ordinaire [...]. Le "lecteur ordinaire" affronte toute situation romanesque comme une affaire personnelle où il est impliqué sur le mode du "comme si" [...]. En fait l'engagement imaginaire dans une autre vie joue de l'écart entre familiarité et étrangeté, connu et inconnu, possible et impossible », G. Mauger et C. F. Poliak, « Les usages sociaux de la lecture ", Genèse de la croyance littéraire, Actes de la recherche en sciences sociales, $\mathrm{n}^{\circ} 123$, juin 1998, Paris, Seuil, p. 6.

14. G. Flaubert, Madame Bovary, ouvr. cité, p. 26.

15. G. Flaubert, Madame Bovary, ouvr. cité, p. 125.

16. G. Flaubert, Madame Bovary, ouvr. cité, p. 69.

17. R. Barthes, «Écrire la lecture » [1970], Le bruissement de la langue, Paris, Seuil, 1984, p. 35-36.

18. Dépliant « Promenade au pays d'Emma Bovary ", office de tourisme des Trois Vallées à Ry. En ligne : <https://cdt76.media.tourinsoft.eu/upload/Depliant-Promenades-au-pays-d-EmmaBovary.pdf> (consulté le 5 janvier 2020).

19. Madame Bovary, ouvr. cité, p. 72.

20. Madame Bovary, ouvr. cité, p. 151. 
21. La politique de la municipalité de Ry en matière de tourisme littéraire est ancienne et s'intensifie aujourd'hui. Les Amis de Flaubert et de Maupassant s'en réjouissent aussi - «La foule montait vers le Comice. Elle allait y assister à la "représentation" de pages éblouissantes de Madame Bovary, mimées par les enfants en costume, sur les textes mêmes de Gustave Flaubert, enregistrés sur bande magnétique et diffusés par haut-parleur. Tous les personnages du grand roman animaient la rue fidèlement restituée et même au trot de ses chevaux, l'Hirondelle qui dévalait la côte! » (J. Le Povremoyne, «Les Comices agricoles de Ry en 1961 », bulletin nº 19, 1961, p. 83).

22. Madame Bovary, ouvr. cité, p. 45.

23. Madame Bovary, ouvr. cité, p. 315.

24. C. Lévi-Strauss, « Le temps retrouvé », La Pensée sauvage, Paris, Plon, 1962, p. 291.

25. Il faut bien reconnaitre que notre romancier aime la blague et même cette forme d'humour ou d'ironie facile qui permet de s'affranchir à moindre frais de l'usage orthodoxe ou sérieux du langage : « Enfin, M. Larivière allait partir, quand $\mathrm{M}^{\mathrm{me}}$ Homais lui demanda une consultation pour son mari. Il s'épaississait le sang à s'endormir chaque soir après le dîner. - Oh! ce n'est pas le sens qui le gêne. Et, souriant un peu de ce calembour inaperçu, le docteur ouvrit la porte", Madame Bovary, ouvr. cité, p. 329.

26. II, chap. 15 : acte I, entrée de Lagardy - brouillons, vol. 5, folio 27v, <https://flaubert.univrouen.fr/jet/public/trans.php?corpus=bovary\&id=3633\&mot=bercement $+\&$ action=MC $>$, (consulté le 5 mai 2020).

27. G. Flaubert, Madame Bovary, ouvr. cité, p. 38.

28. G. Flaubert, Madame Bovary, ouvr. cité, p. 85.

29. II, chap. 2, Conversation Emma - Léon (2), brouillons, vol. 2, folio 60, <https://flaubert.univrouen.fr/jet/public/trans.php?corpus=bovary\&id=3633\&mot=bercement $+\&$ action=MC $>$ (consulté le 5 mai 2020).

30. Ibid.

31. Ibid.

32. Br. vol. 2, folio 63

33. En un sens l'activation littéraire proposée par les lieux du roman entre en homologie avec la libre activité lectrice : « Le lecteur est le producteur de jardins qui miniaturisent et collationnent un monde [...]. Il se déterritorialise [...], tantôt comme le chasseur dans la forêt, il a l'écrit à l'œil [...], tantôt il y perd les sécurités fictives de la réalité », M. de Certeau, "Lire: un braconnage ", L'invention du quotidien, t. 1, dans Arts de faire, Paris, U.G.E, 10/18, 1980, p. 291.

34. J.-M. Privat, « À mots couverts ", Cahiers de littérature orale, 75-76, [2014], 5 mai 2015. En ligne : $<$ https://doi.org/10.4000/clo.2025> (consulté le 16 février 2019).

35. G. Flaubert, Madame Bovary, ouvr. cité. Les citations renvoient respectivement aux pages 17, 82, 116, 339 et 341-342 de notre édition de référence.

36. A.-J. Greimas, «Introduction », Des dieux et des hommes, Paris, PUF, 1985, p. 21. Sur la porosité des frontières du légendaire et du fictif dans les récits de vie extraordinaires, voir A.ClercRenaud et C. Leguy, "Éditorial ", Cahiers de littérature orale $n^{\circ} 80,2016$. En ligne le 13 octobre 2017 : <http://journals.openedition.org/clo/2879> (consulté le 28 mai 2018).

37. Sur Emma comme mythe littéraire, voir par exemple Y. Leclerc, « Comment une petite femme devient mythique », A. Buisine (dir.), Emma Bovary, Paris, Autrement/Figures mythiques, p. 8-25.

38. Pierre Laurence, «La Vieille », p. 226-229. On sait combien rites, croyances et oralité conteuse sont liés dans les cultures d'autrefois, et c'est particulièrement le cas autour de la figure de la Vieille de Carême, vouée par définition à une mort cyclique. P. Laurence, «Entre légendaire fantastique et légendaire toponymique: la Vieille Morte en Cévennes ", Rives nordméditerranéennes, $\mathrm{n}^{\circ}$ 11, [2002], 21 juillet 2005. En ligne: <https://doi.org/10.4000/rives.117> (consulté le 30 septembre 2016). 
39. A. Le Braz, «La légende de la mort chez les Bretons armoricains » [1922], Magies de la Bretagne, Paris, Robert Laffont, 1994, p. 80.

40. H. Hubert et M. Mauss, «Étude sommaire de la représentation du temps dans la religion et la magie ", Mélanges d'histoire des religions, 1905, 122. On observera que «les mythes rajeunis dans l'histoire » ne sont autres que les légendes, même si Arnold van Gennep [La formation des légendes, Paris, Flammarion, 1910, p. 30] s'efforce de distinguer - sans s'y tenir - légende et mythe : "On entendra par légendes un récit localisé, individualisé et objet de croyance, et par mythe une légende en relation avec le monde surnaturel et qui se traduit en actes par des rites.» L'ethnographe consacre un court chapitre à la "littérarisation " des thèmes légendaires (ouvr.cité, p. 216-222). Il trace un programme de recherche qui viserait à comprendre « comment la littérature [romantisme, naturalisme] peut utiliser à ses propres fins des cadres légendaires " et à montrer comment la légende localisée peut devenir « un thème littéraire de portée largement humaine ». André Jolles parle aussi à propos des légendes de la puissance de la figuration de formes simples (modèles prototypiques) sur l'imaginaire, puissance exercée par des "gestes verbaux" issus de la vie réelle et qui interviennent dans cette même vie, quitte à s'actualiser sous des formes spécifiques dans la littérature («La Légende », Formes simples [1930], Paris, Seuil, 1972, p. 27-54).

41. G. Flaubert, Madame Bovary, ouvr. cité, p. 168.

42. G. Flaubert, Madame Bovary, ouvr. cité, p.315-333. Ce destin tragique n'exclut pas bien entendu les songes féeriques d'Emma, jeune. Au début de son initiation à la vie de femme, elle est une Belle au bois dormant qui attend son prince charmant [Juliette Frölich, « Charles Bovary et La Belle au bois dormant », Revue romane, vol. 12, 1997-2, p. 202-209. En ligne : <https://tidsskrift.dk/ revue_romane/article/view/29230/26071> (consulté le 20 février 2020)] et Flaubert lui fera joliment rêver à des « jours beaux comme un ronde de fées » (brouillons, vol. 4, folio 2).

43. «À l'origine d'une légende, il y aurait toujours un criminel ou un saint, dans les deux cas un monstre, celui par qui le scandale arrive, celui qui s'est dressé, en solitaire, pour secouer le joug tyrannique des lois », P. Cordoba, «La Revenance. Pour une pragmatique de la légende », Poétique, $\mathrm{n}^{\circ} 60,1984$, p. $438-445$.

44. La première femme de Charles est veuve. La veuve Dubuc est laide et froide, acariâtre et sèche comme un bâton. C'est une haridelle dont les harnais ne valent pas la peau, selon le père de Charles. Bientôt elle sera prise d'un crachement de sang [...] : «Elle était morte !» (G. Flaubert, Madame Bovary, ouvr. cité, p. 21).

45. G. Flaubert, Madame Bovary, ouvr. cité, p. 69. Le circuit Bovary a été imaginé au départ par des agricultrices locales qui à la fois se retrouvaient en quelque façon dans l'assignation existentielle d'Emma [« on la comprend en un sens »..., déclare l'une d'elles] et souhaitaient s'investir dans une initiative qui valorise leur collectif et leur petit pays (enquêtes de terrain personnelles).

46. C. Fabre-Vassas et D. Fabre, "Une naissance du roman", Du rite au roman, dans Yvonne Verdier, Coutume et destin. Thomas Hardy et autres essais, Paris, Gallimard, 1995, p. 29-34.

47. «J'avais l'intention de ne rapporter ici qu'un simple pèlerinage féminin, mais il n'est peutêtre pas sans intérêt de rappeler qu'on a retrouvé les traces de tous ceux qui furent mêlés au drame. Grâce à la précieuse documentation de l'érudit journaliste rouennais M. Georges Dubosc, ainsi qu'aux recherches curieuses du docteur Raoul Brunon, on sait que l'apothicaire Homais, l'amoureux Justin, Hippolyte le pied-bot et le père Hivert ont vécu [...] », Georgette Leblanc [Mme Maeterlinck], Un pèlerinage au pays de Madame Bovary, Paris, Sansot et Cie, 1913, p. 42.

48. M. Leiris, "Miroirs à pèlerins ", dans Échanges et communications, Mélanges offerts à Claude LéviStrauss réunis par J. Pouillon et P. Maranda, Paris, Mouton, 1970, II, p. 515-518. Repris dans Frêle bruit, Paris, Gallimard, 1976, p. 233. Voir aussi, D. Fabre, ouvr. cité, deuxième partie : Sanctuaires, cultes et pèlerinages.

49. N. Belmont («Croyances et légendes topographiques", Mythes et croyances dans l'ancienne France, Paris, Flammarion, 1973, p. 31-47) rappelle à ce sujet combien « un véritable réseau de 
croyances et de récits légendaires quadrillait littéralement le territoire [...], à l'échelle de ce que pouvaient connaître les habitants d'un village avant la civilisation industrielle. Le schéma de ces récits se retrouve très souvent dans des lieux à la fois précis et différents ".

50. La religion est évidemment très présente dans le roman, y compris dans la trajectoire existentielle et morale d'Emma : «Elle voulut devenir une sainte. Elle acheta des chapelets, elle porta des amulettes; elle souhaitait avoir dans sa chambre, au chevet de sa couche, un reliquaire enchâssé d'émeraudes, pour le baiser tous les soirs. ", G. Flaubert, Madame Bovary, ouvr. cité, p. 219.

51. Les Rogations, qui consistent en trois jours de processions dans les champs, accompagnées de bénédictions et de prières afin d'obtenir d'abondantes récoltes et d'éloigner les fléaux climatiques, présentent quelque analogie avec l'autochtonie du pèlerinage littéraire. En principe l'ambulation couvre tout le terroir marqué de point en point par des croix érigées et parfois décorées pour l'occasion, avec offrandes votives, etc. (Mythes et croyances dans l'ancienne France, ouvr. cité, p. 96-97).

52. La festivalisation du site se développe dans un contexte plus général de revival de la vie villageoise d'antan et de reconstitution historique: "Dans le petit village où certaines façades sont de véritables machines à remonter le temps, des personnages en redingote apparaissent au coin des rues. L'an dernier, à l'initiative du Comité Bovary, un festival avait animé la commune pendant trois jours. La municipalité a décidé de reconduire la manifestation ce week-end intitulée « Bourg de Ry ». En ligne : 〈https://www.paris-normandie.fr/region/ry-plonge-dans-lemonde-de-flaubert-DD12886533> (consulté le 19 janvier 2020). Ou encore : « Le long de la grande rue, dix panneaux ont été installés à proximité des lieux emblématiques du roman tels que la maison des Delamare, l'étude de maître Guillaumin, la pharmacie Homais, etc. Chaque borne présente un extrait du roman, une explication du lien entre Ry et le village d'Yonville inventé par Flaubert [...]. Un flash-code ludique permet également d'accéder par smartphone à des extraits audio et des contenus supplémentaires (traduits en anglais), qui plongeront le visiteur dans l'univers de Mme Bovary. » En ligne : <http://www.seineetbray.fr/page,1,49,235.html> (lien désormais inactif, consulté le 19 janvier 2020). Ou même: "Le lundi, nous proposons une randonnée de $10 \mathrm{~km}$ au cours de laquelle nous suivons le parcours d'Emma lorsqu'elle rend visite à ses amants : Rodolphe, un séducteur qu'Emma rencontre aux comices agricoles et le jeune Léon, clerc de notaire. Encadrée par un guide, cette visite plonge au cœur du roman mais permet aussi de découvrir le patrimoine local comme la chapelle privée Saint-Denis-le-Thiboult. » En ligne : <https://www.tendanceouest.com/actualite-223581-festival-bovary-a-ry.html> (consulté le 19 janvier 2020).

53. M. Halbwachs, "La mémoire collective et l'espace ", La mémoire collective [1950], Paris, PUF, 1968, p. 160-167.

54. E. Auerbach, Mimésis. La représentation de la réalité dans la littérature occidentale [1946], Paris, Gallimard/TEL, 1968, p. 550.

55. M. Halbwachs, La Topographie légendaire des évangiles en Terre sainte [1941], Paris, PUF, 2017, p. 123-124.

56. Ibid., p. 130-131.

57. "Ry et Madame Bovary", site terres d'écrivains, 28 août 2003. Voir en ligne : <http:// www.terresdecrivains.com/Ry-et-Madame-Bovary> (consulté le 19 janvier 2020).

58. R. Barthes, «Sur la lecture », Le bruissement de la langue, ouvr. cité, p. 46.

59. G. Flaubert, Madame Bovary, ouvr. cité, p. 59. 


\section{RÉSUMÉS}

Un lieu (un petit village), un personnage (Emma B.), un dispositif (un site littéraire). L'étude ethnographique et sémiotique analyse comment un personnage de papier au destin (trop) banal et tragique a été promu au rôle de fiction vivante. Ce transfert de fiction romanesque se déploie sous forme d'une scénographie d'automates miniatures - La Galerie Bovary - et s'offre à un parcours dans l'espace concret d'un terroir normand - Promenade au pays d'Emma Bovary. Ce tourisme de loisir et de bonne volonté culturelle ignore délibérément la textualité du roman de Flaubert au profit d'un jeu/je de feintise ludique avec l'héroöne de la funeste romance. L'analyse vise à montrer comment des types de médiation à la littérature peuvent s'inscrire dans une longue durée culturelle (oralité folklorique topique et créance dans le légendaire chrétien), en ses formes et valeurs contemporaines.

A place (a small village), a character (Emma B.), a device (a literary location). This ethnographic and semiotic study analyzes the way in which a storybook character with a (very) banal and tragic destiny has been promoted to the rank of a living fiction. This adaptation of a fictional character comes to life in the form of a scene involving miniature automats - La Galerie Bovary and presents itself in the form of an itinerary in the real space of the Norman countryside $-A$ Promenade in the Country of Emma Bovary. This well-intentioned tourist attraction does not make any attempt to evoke the text of Flaubert's novel, preferring to play on and with the heroine of the grim romance. This study aims to demonstrate how forms of literary mediation can be seen as part of a long-term cultural history (oral folklore themes and belief in Christian legends) seen from the point of view of its contemporary values and forms.

\section{INDEX}

Mots-clés : site littéraire, Madame Bovary, fiction vivante, légende topique, transfert de sacralité

Keywords : literary location, Madame Bovary, living fiction, common legend, transfer of sacredness

\section{AUTEUR}

\section{JEAN-MARIE PRIVAT}

Jean-Marie Privat est professeur émérite en langue et littérature françaises, spécialiste d'ethnocritique. Il s'intéresse particulièrement à l'anthropologie de l'écrit et aux imaginaires culturels. Pour ses travaux et publications en ethnocritique, voir le site $<$ www.ethnocritique.com>. 